\title{
Immediate and late benefits of treating very elderly people with hypertension: results from active treatment extension to Hypertension in the Very Elderly randomised controlled trial
}

\author{
(c) $\underset{1}{(1)(8)}$ OPEN ACCESS
}

N Beckett honorary clinical senior lecturer ${ }^{1}$, R Peters science and business development manager ${ }^{2}$, $\mathrm{J}$ Tuomilehto professor of public health ${ }^{34}$, C Swift professor of health care of the elderly ${ }^{5}$, P Sever professor of clinical pharmacology and therapeutics ${ }^{6}$, J Potter professor of ageing and stroke medicine $^{7}, \mathrm{~T}$ McCormack general practitioner ${ }^{8}$, $\mathrm{F}$ Forette professor of internal medicine and geriatrics $^{9}$, B Gil-Extremera professor for internal medicine ${ }^{10}$, D Dumitrascu professor of medicine ${ }^{11}$, $\mathrm{J}$ A Staessen head of Studies Coordinating Centre ${ }^{12}$, L Thijs statistician ${ }^{13}$, A Fletcher professor of epidemiology of ageing ${ }^{14}$, C Bulpitt emeritus professor of geriatric and cardiovascular medicine ${ }^{1}$, for the HYVET Study Group

\footnotetext{
${ }^{1}$ Department of Medicine, Imperial College London, London SW7 2AZ, UK; ${ }^{2}$ Clinical Trials Unit, Imperial College London; ${ }^{3}$ Department of Public Health, University of Helsinki, Helsinki, Finland; ${ }^{4}$ South Ostrobothnia Central Hospital, Seinäjoki, Finland; ${ }^{5}$ Clinical Age Research Unit, Department of Clinical Gerontology, Kings College London, London; ${ }^{6}$ International Centre for Circulatory Health, Imperial College London; ${ }^{7}$ School of Medicine, Health Policy and Practice, University of East Anglia, Norfolk, UK; ${ }^{8}$ Whitby Group Practice, Spring Vale Medical Centre, Whitby, UK; ${ }^{9} \mathrm{Hôpital} \mathrm{Broca,}$ University Paris V, Paris, France; ${ }^{10}$ FAC Medicina, Departmento Medicina Granada, Granada, Spain; ${ }^{11}$ Spitalul Judetean Cluj, Clinica Medicală 2 , Cluj, Romania; ${ }^{12}$ Department of Epidemiology, Maastricht University, Netherlands; ${ }^{13}$ Studies Coordinating Centre, Division of Hypertension and Cardiovascular Rehabilitation, Department of Cardiovascular Diseases, University of Leuven, Leuven, Belgium; ${ }^{14}$ London School of Hygiene and Tropical Medicine, London
}

\begin{abstract}
Objective To assess if very elderly people with hypertension obtain early benefit from antihypertensive treatment.

Design One year open label active treatment extension of randomised controlled trial (Hypertension in the Very Elderly Trial (HYVET)).

Setting Hospital and general practice based centres mainly in eastern and western Europe, China, and Tunisia.

Participants People on double blind treatment at the end of HYVET were eligible to enter the extension.

Interventions Participants on active blood pressure lowering treatment continued taking active drug; those on placebo were given active blood pressure lowering treatment. The treatment regimen was as used in the main trial-indapamide SR $1.5 \mathrm{mg}$ (plus perindopril 2-4 mg if required) - with the same target blood pressure of less than $150 / 80 \mathrm{~mm}$ $\mathrm{Hg}$.
\end{abstract}

Main outcome measures The primary outcome was all stroke; other outcomes included total mortality, cardiovascular mortality, and cardiovascular events.

Results Of 1882 people eligible for entry to the extension, 1712 (91\%) agreed to participate. During the extension period, 1682 patient years were accrued. By six months, the difference in blood pressure between the two groups was $1.2 / 0.7 \mathrm{~mm} \mathrm{Hg}$. Comparing people previously treated with active drug and those previously on placebo, no significant differences were seen for stroke ( $n=13$; hazard ratio 1.92, 95\% confidence interval 0.59 to 6.22$)$ or cardiovascular events $(n=25 ; 0.78$, 0.36 to 1.72 ). Differences were seen for total mortality ( 47 deaths; hazard ratio $0.48,0.26$ to $0.87 ; \mathrm{P}=0.02$ ) and cardiovascular mortality (11 deaths; $0.19,0.04$ to $0.87 ; P=0.03$ ).

Conclusion Very elderly patients with hypertension may gain immediate benefit from treatment. Sustained differences in reductions of total mortality and cardiovascular mortality reinforce the benefits and support the need for early and long term treatment.

Trial registration Clinical trials NCT00122811. 


\section{Introduction}

Hypertension remains a risk factor for cardiovascular mortality and morbidity in very elderly people. ${ }^{1}$ However, before the results of the Hypertension in the Very Elderly Trial (HYVET), uncertainty existed about the benefits and risks associated with the treatment of very elderly people with hypertension. ${ }^{2}{ }^{3}$ HYVET was stopped early by the independent Trial Steering Committee and Data Monitoring Committee owing to evidence of a reduction in mortality with active treatment. Despite the short follow-up during the main trial, with a median follow-up of 1.8 years (mean 2.1 years), clear evidence of benefit from active treatment was found. Total mortality was reduced by $21 \%$ (95\% confidence interval $4 \%$ to $35 \% ; \mathrm{P}=0.019)$ and cardiovascular events (cardiovascular mortality, non-fatal myocardial infarction, non-fatal stroke, and non-fatal heart failure) by $34 \%$ ( $18 \%$ to $47 \%$; $\mathrm{P}<0.001) .{ }^{4}$ No evidence of an adverse effect from the treatment used in the trial was seen; significantly fewer serious adverse events occurred with active treatment, and no suggestion of a deterioration in cognitive function or an increase in fractures was seen. ${ }^{56}$

Extended follow-up in the Syst-Eur trial suggested that earlier intervention provided greater benefit in younger elderly people with hypertension. ${ }^{7}$ The HYVET Steering Committee agreed that a one year open label extension to run seamlessly from the end of the main trial might provide additional information to that obtained during the main trial. In particular, people speculated whether early benefits would be seen in such an elderly group. Additional safety information would also be obtained. We report here the main findings from the trial extension.

\section{Methods}

The detailed protocol for HYVET has been published. ${ }^{8}{ }^{9}$ In brief, it was a randomised, double blind, placebo controlled trial in 195 centres in 13 countries in western and eastern Europe, China, Australasia, and Tunisia.

To be eligible for entry into the main trial, participants had to be aged 80 years or over with sustained levels of systolic blood pressure of $160 \mathrm{~mm} \mathrm{Hg}$ or above over a two month placebo run-in phase. The independent trial Steering Committee terminated the main trial in July 2007 for ethical reasons, as the independent Data Monitoring Committee noted a significant reduction in all cause mortality in participants on active treatment at the second interim analysis. All final visits related to the main trial were completed by 12 October 2007 . The final visit was counted as the entry visit to the extension.

To be eligible for entry into the extension, participants simply needed to be on double blind treatment at the time of their final visit. No other exclusion criteria for the extension were considered. During the main trial, participants who reached the primary or secondary end points (apart from myocardial infarction, heart failure, and skeletal fracture) were withdrawn from double blind treatment; even if they were followed up on open label drug after the event, they would not have been eligible for the extension. In the end, no participant who entered the extension had reached an end point during the main trial.

At the start of the trial, blood pressures were recorded with either a mercury sphygmomanometer or a validated automated device; by the end, most centres were using an automated device. At the start of the trial in 2000, the average seated diastolic blood pressure had to be $90-109 \mathrm{~mm} \mathrm{Hg}$, but in 2003 a protocol amendment relaxed this criterion to less than $110 \mathrm{~mm} \mathrm{Hg}$, allowing the inclusion of patients with isolated systolic hypertension; the standing systolic blood pressure criterion remained the same at $140 \mathrm{~mm} \mathrm{Hg}$ or above.

The treatment steps used in the main trial were indapamide SR $1.5 \mathrm{mg}$ alone (step I), then indapamide SR plus perindopril 2 $\mathrm{mg}$ (step II), and finally indapamide SR plus perindopril $4 \mathrm{mg}$ (step III) (or corresponding matching placebos). At the start of the one year extension, all participants were restarted at the first step irrespective of the level of treatment under double blind follow-up. At the time of the start of the extension and for four months afterwards (until the freezing of the database for the main trial), the coordinating centre was not supplied with the code to break the blind, so local investigators were not informed whether participants had previously been on active or placebo drug. Titration of trial drug was encouraged to achieve the goal of systolic blood pressure below $150 \mathrm{~mm} \mathrm{Hg}$ and diastolic blood pressure below $80 \mathrm{~mm} \mathrm{Hg}$. The use of additional antihypertensive agents at the discretion of the local investigator was allowed if the target was not achieved, but only in the extension.

Participants provided written informed consent before starting the extension. For those who were illiterate, an independent witness signed the consent form.

\section{Data collection}

Baseline characteristics were collected during the placebo run-in period. After randomisation, participants were seen at least every three months during the first year and at least every six months thereafter. During the one year extension, visits took place every three months. At the end of the double blind phase and at the end of the one year extension, we collected information on current diseases, drug treatment, blood pressure, biochemistry (sodium, potassium, urea, creatinine, glucose, uric acid) cholesterol (total and high density lipoprotein), and haematology (haemoglobin, haematocrit). At three and six monthly visits, only information on current diseases, drug treatment, and blood pressure was collected. All final visits for the one year extension were completed by 12 October 2008 .

\section{End points}

During the extension, the same endpoint data were collected as during the main trial. These included all strokes (fatal and non-fatal, but not transient ischaemic attacks), total mortality, cardiovascular mortality, cardiac mortality, and mortality from stroke. An independent committee blinded to previous treatment allocation reviewed all possible end points by using predefined definitions as stated in the protocol. ${ }^{8}$ Events were classified as cardiovascular or non-cardiovascular. Cardiac mortality included fatal myocardial infarction, fatal heart failure, and sudden death. Cardiovascular events consisted of cardiovascular mortality, non-fatal myocardial infarction, non-fatal stroke, and non-fatal heart failure.

\section{Data monitoring and statistical analysis}

The same independent data monitoring committee oversaw the one year extension. The sample size for the original trial was powered on the primary end point of all strokes. ${ }^{8}$ As the main trial stopped early and all participants returned for final visits, consent for participation in an extension could be obtained at this visit. This maximised the potential for including as many patients as possible for further follow-up. At this time, final data were being collected and cleaned, so the exact rates and differences between the two original groups were not known. The code for breaking of the blind was not provided to the coordinating centre until five months after the final visits, so 
we could not do power calculations on the basis of the collected data. Given this, and that the existing trial was to run seamlessly into the extension, we did not do revised power calculations.

No further follow-up data were collected on participants on open label drug at the time of the final main trial visits. As only those participants who were on double blind treatment were recruited into the one year extension, we did not consider it appropriate to combine the data from the one year extension with that from the main trial. The data from the one year extension were thus considered in isolation, comparing participants previously on active treatment with those previously on placebo treatment.

We calculated patient years from the date of starting active treatment in the one year extension until death or the last available visit. Participants were free to withdraw from the extension should they wish, but no protocol defined withdrawal events existed for the extension.

We compared means of continuous variables by $\mathrm{z}$ test, proportions by $\chi^{2}$ test, and incidence rates by log rank test. We used the Kaplan-Meier method to estimate cumulative incidence curves. We took a $\mathrm{P}$ value of 0.05 to be significant. For participants with more than one end point, we used time to the first relevant end point in each analysis. We adjusted results for all stroke, total, and cardiovascular mortality for sex, age, baseline seated systolic blood pressure, and cardiovascular disease before randomisation to the main trial. We calculated hazard ratios with a Cox proportional model. We checked the proportional hazard assumption by plotting the Schoenfeld residuals. All reported $\mathrm{P}$ values are two sided and not adjusted for multiple testing. We used SAS version 9.1.3 for data management and analyses.

\section{Results}

At the end of the main HYVET trial, 1882 participants were on double blind treatment. Of these, 1009 were receiving active trial treatment and 873 were receiving placebo (fig $1 \Downarrow$ ). Of these, $1712(91 \%)$ consented to enter the one year extension-924 $(54 \%)$ of these were taking active treatment and 788 (46\%) were taking placebo. The participation rates were similar at $91.6 \%$ for active treatment and $90.3 \%$ for placebo. No additional information was collected on the 170 participants (85 in each group) who did not consent to enter the open label extension or the 220 participants who were on open label treatment at the end of the main trial.

Participants who entered the extension were mostly from eastern Europe $(n=493)$ and China $(n=1144)$. The rest were from western Europe, Australasia, or Tunisia. The participation rates were similar in most countries, ranging from $73 \%$ to $96 \%$.

Table $1 \Downarrow$ shows the characteristics of participants who did and did not enter the one year extension, at the time of entry. Those who entered the extension were significantly younger than those who did not, and they had higher systolic blood pressure, lower total cholesterol, and lower serum creatinine at the end of the main trial. The biochemical differences are likely to reflect the higher participation by Chinese people. Compared with the baseline characteristics at the start of the main trial, ${ }^{4}$ people entering the extension were on average 1.5 years older with slightly higher serum creatinine; they had lower average blood pressure and body mass index and a lower prevalence of orthostatic hypotension, diabetes mellitus, and smoking but similar heart rate, serum uric acid concentration, and total cholesterol and high density lipoprotein cholesterol.
Among participants who entered the extension, 235 (25\%) of those receiving active treatment were on indapamide alone (step I), 262 (28\%) were on indapamide SR and perindopril $2 \mathrm{mg}$ (step II), and 416 (45\%) were on indapamide SR and perindopril $4 \mathrm{mg}$ (step III) at the end of the double blind phase. The corresponding numbers for the placebo group were $129(16 \%)$, $114(14 \%)$, and $538(68 \%)$. All patients were restarted on indapamide alone at the start of the extension, irrespective of what they were taking at the end of the double blind phase. By six months, little difference existed in the number at any particular treatment step between participants previously taking active drug and those previously taking placebo. Of those previously taking active drug, 239 (27\%), 290 (33\%), and 346 (39\%) were at steps I, II, and III compared with 183 (25\%), 212 (28\%), and $346(46 \%)$ of those previously taking placebo. At the end of the one year extension, the numbers were also similar-213 (25\%), $182(21 \%)$, and $465(53 \%)$ for those previously on active treatment and $160(22 \%), 149(21 \%)$, and $412(57 \%)$ for those previously taking placebo. Although additional blood pressure lowering treatment was allowed in the extension to reach the target pressure, the number taking such drugs in addition to the trial drugs was small at $16(0.9 \%)$ at six months and $31(1.8 \%)$ after one year. Adherence to trial drugs was high on the basis of the reported number of days that drug was missed, with an overall rate of adherence of $99 \%$.

At the end of the double blind phase, the mean sitting blood pressure was $145.0 / 76.6 \mathrm{~mm} \mathrm{Hg}$ in participants on active drug who went into the extension and 159.3/80.8 $\mathrm{mm} \mathrm{Hg}$ in those on placebo $(\mathrm{P}<0.001)$, a difference of $14.3 / 4.2 \mathrm{~mm} \mathrm{Hg}$. By six months, the difference in blood pressure was not statistically different between those previously on active treatment and those previously on placebo $(1.3 / 0.6 \mathrm{~mm} \mathrm{Hg})$; the mean blood pressures in the groups were 145.3/76 $\mathrm{mm} \mathrm{Hg}$ and 146.6/76.6 $\mathrm{mm} \mathrm{Hg}$ (fig $2 \Downarrow$ ). This difference remained minimal until the end of the extension, when the blood pressure was 142.8/76.2 $\mathrm{mm} \mathrm{Hg}$ in participants previously on active drug and 143.8/76.4 $\mathrm{mm} \mathrm{Hg}$ in those previously on placebo, a difference of 1.0/0.2 $\mathrm{mm} \mathrm{Hg}$.

During the one year extension, 47 participants died (11 of the deaths were considered to be cardiovascular) and 44 withdrew from follow-up. Two patients were considered lost to follow-up, and we censored their data at the time of their last visit. At the end of follow-up, 1619 participants were alive and 1682 patient years of follow-up had been accrued.

For the primary end point (fatal and non-fatal stroke), 13 events occurred during the extension, with no significant difference between participants previously on active treatment and those previously on placebo (hazard ratio $1.92,95 \%$ confidence interval 0.59 to $6.22 ; \mathrm{P}=0.28$ ) (table $2 \Downarrow$ ). Similarly, we found no differences between the two groups for heart failure events (hazard ratio $0.28,0.03$ to $2.73 ; \mathrm{P}=0.28$ ) or the composite end point of cardiovascular events $(0.78,0.36$ to $1.72 ; \mathrm{P}=0.55)$. We found differences in total mortality (hazard ratio $0.48,0.26$ to $0.87 ; \mathrm{P}=0.02)$ and cardiovascular mortality $(0.19,0.04$ to 0.87 ; $\mathrm{P}=0.03$ ). Adjustment for age, sex, baseline sitting systolic blood pressure, and previous cardiovascular disease did not change the results.

The local investigators did not report any serious adverse drug reactions during the extension period. In total, 99 serious adverse events were reported-53 in participants previously treated with placebo and 46 in those previously on active treatment $(\mathrm{P}=0.12)$. The mean biochemical changes over the one year extension were all small and not clinically significant in the two groups. We noted statistical differences between the two groups for serum sodium (mean decrease $0.6 \mathrm{mmol} / \mathrm{L}$ for placebo and 0.1 
$\mathrm{mmol} / \mathrm{L}$ for active treatment; $\mathrm{P}=0.04)$ and for serum uric acid (mean increase $15.5 \mu \mathrm{mol} / \mathrm{L}$ for placebo and $0.7 \mu \mathrm{mol} / \mathrm{L}$ for active treatment; $\mathrm{P}=0.001$ ).

Comparing the results for the extension against those for the main trial, we noted that the rates per 1000 patient years were lower in the extension than in the main trial irrespective of whether participants had previously been treated with active or placebo drug (fig $3 \Downarrow$ ). For stroke, the rate during the main trial was 11.7 per 1000 patient years in the active treatment group and 17.3 per 1000 patient years in the placebo group. The rates during the extension were 9.9 per 1000 patient years for participants previously on active treatment and 5.2 per 1000 patient years for those previously on placebo. The difference during the main trial was significant $(\mathrm{P}=0.04)$, unlike during the extension. Although significant differences in total mortality remained during the extension, the rates were lower than during the main trial.

\section{Discussion}

The results of the one year open label extension have added to those of the main HYVET trial. The lack of difference in incidence of heart failure and stroke between participants previously receiving active treatment and those previously receiving placebo suggests that early benefits can be achieved from treating and controlling blood pressure in very elderly people with hypertension with the treatment used in the trial. The fact that differences remained in all cause mortality and cardiovascular mortality supports the idea that some benefits from control of blood pressure take longer to accrue and that early treatment is thus warranted.

Even at the age of 80 , average life expectancy can be around seven years for men and nine years for women. ${ }^{10}$ Considering the prevention of adverse cardiovascular events even at this advanced age is thus appropriate, as a reduction in disability and maintenance of independence and quality of life would clearly be of benefit. In HYVET, most participants initially recruited to the trial did not have established cardiovascular disease. Participants entering the extension had not reached a cardiovascular end point during the main trial, so the results of both studies are most applicable to preventing cardiovascular events in people free of established cardiovascular disease. The fact that the differences in cardiovascular events overall between active treatment and placebo seen in the main trial were lost within one year of active antihypertensive treatment reinforces the suggestion that the over 80 s should be screened regularly with a view to treatment if they have sustained blood pressures of $160 \mathrm{~mm} \mathrm{Hg}$ or above as they are likely to gain benefits from early detection.

The difference in blood pressure that existed at the start of the extension was essentially abolished within six months. As in the active arm of the trial, most participants (approximately three quarters) needed combination therapy of indapamide SR and perindopril at either the $2 \mathrm{mg}$ or $4 \mathrm{mg}$ dosage. Additional non-trial drugs were needed in only a small number of participants. The overall achieved mean blood pressure at six months was 146/76 mm Hg, suggesting that the achievement of blood pressures below $150 / 80 \mathrm{~mm} \mathrm{Hg}$ is possible in most very elderly people and that such combination treatment is effective. These results do not answer the question of whether even lower achieved pressures would be more effective in this age group, and additional research is needed to determine this. We saw no suggestion of an increase in serious adverse events to suggest detrimental effects from such an achieved reduction in blood pressure. In addition, the minor non-clinically important biochemical changes seen during the extension support the use of a combination of sustained release indapamide and low dose perindopril in such elderly patients with hypertension.

Treatment of hypertension is considered to be life long. Most clinical trials are of short duration, however, as they have been designed to test the trial hypothesis within a certain period of time and powered to do so. Stopping the HYVET trial early was entirely appropriate, given the clear benefits that were seen. The additional patient years accrued during the extension extended the overall exposure to treatment, especially among participants who were on active drug from the start of the trial. Given that the event rates were lower in the extension than in the main trial, this adds weight to the importance of long term antihypertensive treatment in very elderly people with high blood pressure. However, people who entered the extension, despite being on average older than those at entry to the main trial, seemed to be healthier with lower rates of diabetes and smoking and lower average blood pressures, and this may have led to lower event rates. Despite this, differences in total mortality and cardiovascular mortality remained during the extension, indicating that reductions in these major end points are likely to take a longer time to accrue. This was already seen in the main trial period, with the divergence in event rates between the randomisation groups gradually increasing over time. ${ }^{4}$ Thus, although this seems to be a later benefit from treatment, it confirms the importance of early and sustained antihypertensive treatment even in very elderly people.

\section{Comparison with other studies}

Other intervention trials in hypertension have also reassessed the long term outcomes of participants originally included in the trial. In a 14.3 year follow-up of participants from the Systolic Hypertension in Elderly Persons (SHEP) trial, cardiovascular mortality was $15 \%$ lower in the original diuretic treated group than in the group originally treated with placebo. ${ }^{11}$ Interestingly, the diuretic treated group were more likely to develop diabetes than were the placebo group (13\% v 8.7\%) The patients with diabetes ascertained in the diuretic treated group had no increase in cardiovascular mortality or all cause mortality during this follow-up period compared with those diuretic treated patients who did not develop diabetes during the trial. No significant changes in blood glucose occurred in the extension of HYVET, in keeping with the result during the main trial. In a separate analysis of a subgroup of the SHEP participants, again followed up for 14 years, patients originally assigned to active treatment had a $90 \%$ lower mortality or incidence of cardiovascular events compared with patients originally assigned to placebo. ${ }^{12}$ The long term benefit from active treatment was greatest in those patients without baseline evidence of atherosclerosis, whereas the benefit over placebo seemed to dissipate in those with baseline evidence of atherosclerosis. Such extended length of follow-up would not be relevant for participants recruited to HYVET, given the average life expectancy. What is most important in octogenarians with hypertension is that any benefits from intervention are achieved as soon as possible. Nevertheless, confirming the longer term sustained benefits from early active antihypertensive drug treatment among very elderly patients is also relevant.

The results of the HYVET extension are also in line with those from the extended follow-up of participants in the Systolic hypertension in Europe trial (Syst-Eur). ${ }^{7}$ The Syst-Eur trial was terminated early and went on to have an open label active extension that increased the median follow-up from 2 years to 6.1 years. Participants were followed up irrespective of whether 
they were on double blind treatment or in open label follow-up when the main trial was terminated. This allowed combination of the extension and the main trial data, unlike in the HYVET extension. The results from the Syst-Eur extension showed that immediate treatment compared with delayed treatment reduced the occurrence of stroke by $28 \%$ and cardiovascular complications by $15 \%$.

\section{Strength and limitations}

One of the main limitations of the HYVET extension was that only participants who were on double blind treatment were enrolled from the main trial. Differential survival between those on active and placebo drugs and withdrawal rates in the main trial meant that the numbers in each group who remained on double blind treatment at the end of the main trial were not balanced. Although participants who had received active treatment during the main trial had similar levels of diabetes, smoking, and cholesterol, they differed from those who had received placebo in that they had higher blood pressures and concentrations of uric acid at the start of the extension period. The exclusion of patients moved to open label treatment during the main trial, with no possibility of further information being obtained if they reached any end points, meant that an analysis of all participants from the time of randomisation was not appropriate. Also, comparing the extension results directly with the main trial results would not be possible. In any case, combining the data obtained in the extension with those from the main trial would simply dilute the results seen in the main trial, as no patient who entered the extension had an end point before that time. Another limitation is the short length of follow-up in the extension. Unfortunately, we did not have resources to do a further long term follow-up among this cohort of patients. Finally, given the small number of events in the extension, caution must be exercised in drawing firm conclusions simply from the extension. The results should be viewed in the context of those from the main trial as well.

\section{Applicability and generalisability}

All octogenarians, irrespective of their blood pressure, are at high risk of a cardiovascular event owing to their age alone. That does not mean that all would benefit from antihypertensive treatment. These results support the treatment of very elderly people with blood pressures over $160 \mathrm{~mm} \mathrm{Hg}$ who do not have cognitive impairment and are not considered to be frail. Further research is needed before clearer recommendations can be proposed for other people. Further research is also needed into whether lowering systolic blood pressure to below $150 \mathrm{~mm} \mathrm{Hg}$ in people aged 80 or more is truly beneficial.

In summary, the results from the extension show that the benefit of the antihypertensive treatment used in HYVET may be accrued within 12 months even in very elderly people for events that are very dependent on blood pressure, such as stroke and heart failure. Benefits in terms of a reduction in mortality and cardiovascular mortality are likely to take longer to accrue. People aged 80 or more should have their blood pressure checked regularly, and if they have sustained levels of $160 \mathrm{~mm}$ $\mathrm{Hg}$ or more should be treated in line with the HYVET regimen with a view to reducing their systolic blood pressure to below $150 \mathrm{~mm} \mathrm{Hg}$.

The committee members and investigators for HYVET were as follows. Coordinating Centre: C J Bulpitt (lead investigator), A E Fletcher (co-investigator), N S Beckett (trial coordinator), R Peters (deputy trial coordinator), HYVET coordinating team at Imperial College London (1999 to 2008).

Steering Committee: T McCormack, J Potter, B G Extremera, P Sever, F Forette, D Dumitrascu, C Swift, J Tuomilehto, J Coope (retired in 2001), C Nachev (deceased).

Data Monitoring Committee: J Staessen, L Thijs, R Clarke, K Narkiewicz. End Points Committee: C Davidson (retired in 2003), J Duggan, G Leonetti, N Gainsborough, M C De Vernejoul, J Wang, V Stoyanovsky.

Dementia Validation Committee: J Tuomilehto, R Clarke, A Waldman, I Walton, C Ritchie.

Ethics Committee: R Fagard, J Grimley Evans, B Williams.

Investigators ("national coordinators): Australia-R Warne*, I Puddey*, M Woodward, R Penhall, C Inderjeeth, S Roger, R Scholes, C Johnson; Belgium-H Celis*, G Adriaens, W Onsea, K Cornelli, D Vantroyen, P Cleen, P de Voogt; Bulgaria-C Nachev* (deceased) (national coordinator from 1998 to 2005), V Stoyanovsky* (national coordinator after 2005), P Solakov, R Prokopova, E Mantova, D Smilkova, S Mantov, K Yankulova, R Kermova, D Popov, V Sirakova, V Gergova, D Kamenova, F Grigorov, T Vassileva, R Alahverdian, M Tzekova, A Postadjian, M Geneva, V Mincheva, T Petrusheva, A Toncheva, I Gruev, V Tsanova; China-L Liu*, H Ge, S Wang, J Wang, W Zhang, S Jin, L Ge, Y F Lu, S Ma, L Shen, J Guo, Z Lv (deceased), R Huang, X Li, B Guo, G E Yuan, T Zhang, L Zhang, J Feng, Z He, J Wang, L Deng, L Liu, Q Yuan, F Zhang, H Li, D Wang, K Yang, M Sun, H Liu, X Yan, F Ren, J Tang, M Zhao, X Luo, H Zhou, H Sang, Jie Wang, L Yan, Zhixing Wang, J Zhang, Chengzhi Wang; Finland-R Antikainen*, T Strandberg, T Konttila, A Hynninen, M Jääskivi, J Airas, T Jääskeläinen, J Tuomilehto, H Litmanen, T Karhi, H Yliharsila; France-F Forette*, J Doucet, J Belmin, A Benetos, G Berrut, T Boge, M Bonnefoy, A Carre, N Charasz, J Covillard, T Dantoine, M Escande, Y Frances, R Joire, C Jeandel, S Legrain, A Lion, M Maillet-Vioud, J P Escaillas, S Meaume, P Pfitzenmeyer, F Puisieux, E Quercy, O Rodat, J Soubeyrand, B de Wazieres, H Hindennach, L Lugassy, J Rossi, M Martel, J-M Paladel, C Ravier, A Visconti, J P Gallet, D Zygouritsas, D Charles, F Flamand, G Grandmottet, M Grandmottetegermann, C Gevrey, P L Mesnier, G Robert, C Besset-Prat, A Brousse, P Lafont, J Morelli, P Vernede, A Volkmann, X Bodin, B Destrube, R Eoche, A Boye, F Seropian, P Gernigon, D Meker, J Thomere, Y Thual, F Volny, E Grassart, M Herent, D Lejay, J-P Lopez, B Mannessier, G Pruvost, J-C Urbina; Ireland-J Duggan*; New Zealand-C Anderson*, S Lillis, J Gommans; H Senior Poland-T Grodzicki* , Z Chodorowski, Z Gaciong; Romania-D Dumitrascu*, M Comsa, V Sandru, G Prada, M Dunca-Moisin, D Jianu, D Jinga-Lazar, V Enachescu, C Zaharia; Russia-Y Nikitin*, A Kirichenko, L Olbinskaya, A Martynov, V Zadionchenko, V Moiseev, G Storohzakov, S Nedogoda, R S Karpov, O Barbarash, G Efremushkin, V Kostenko, M Boyarkin, S Churina, T Tyurina, M Ballyuzek, L Ermoshkina, A Timofeev, S Yakusheva, N Shilkina, V Barbarich, L Latunceva, S Burakova, T Ripp, S Pekarsky, V Mordovin; Tunisia-A Belhani*, E Boughzela, S Soraya, B Youssef-Zouari, A B Khalfallah, M H Houman, A K Abida; United Kingdom-C Rajkumar*, M Wilkins, N D Pandita-Gunawardena, J Potter, E Ekpo, M Price, N de Kare-Silver, A Starczewski, S Chandran, N Nasar, M Datta-Chaudhuri, T McCormack, N Majmudar, A Gordon, L Brawn, T Solanki, F Dockery, R Schiff.

We acknowledge the work of $\mathrm{C}$ Nachev (Steering Committee member, National Coordinator for Bulgaria, and HYVET investigator from 1998 until his death in 2005).

Contributors: $\mathrm{CB}$ and AF designed the trial. The coordinating centre at Imperial College collected the data, which were analysed by RP and LT. NB wrote the first draft of the paper. JT, CS, JP, TMcC, FF, DD, and BG-E, were all members of the Steering Committee that supported and assisted in the concept of the trial extension and all provided intellectual critique of the manuscript. JS chaired the Data Monitoring Committee, was involved in the original design of the main trial, and provided critique on the analysis of the extension. CB is the guarantor. 


\section{What is already known on this topic}

People aged 80 or more with sustained systolic blood pressures of $160 \mathrm{~mm} \mathrm{Hg}$ or more benefit from blood pressure lowering treatment

\section{What this study adds}

Benefits in terms of a reduction in cardiovascular events appeared within one year of starting treatment

The benefit was achieved by lowering systolic blood pressure to $150 \mathrm{~mm} \mathrm{Hg}$; any benefit of lowering blood pressure further cannot be assumed

Free living, community based octogenarians should be screened for hypertension and offered blood pressure lowering treatment in line with HYVET

Sponsors: The trial was coordinated by the Department of Care of the Elderly, Imperial College London. Imperial College was the sponsor of the trial and required confidentiality agreements with all committee members and investigators.

Funding: HYVET was funded by grants from the British Heart Foundation and the Institute de Recherches Internationales Servier. The analysis, interpretation of the data, generation of the manuscript, and decision to submit for publication were carried out independently of the funding bodies.

Competing interests: All authors have completed the Unified Competing Interest form at www.icmje.org/coi_disclosure.pdf (available on request from the corresponding author) and declare: for the submitted work, NB and RP received salary support from a grant made to Imperial College London by the Institute de Recherches Internationales Servier, CB received consultancy fees from the grant from 2005, and the other co-authors received reimbursement for travel costs from the grant; NB has received honorariums from Servier; $\mathrm{CB}, \mathrm{JP}$, and TMcC have received honorariums from various companies that manufacture antihypertensive medications; JP sits on an advisory board for MSD and Bayer; TMcC does consultancy for Servier, Daichii Sankyo, Boehringer Ingelheim, and Takeday, has received travel expenses from Boehringer Ingelheim and MSD, and holds shares in Astra Zeneca; no other relationships or activities that could appear to have influenced the submitted work.

Ethical approval: Approval for the trial and the one year active open label extension, the last by a protocol amendment to the main trial protocol, was obtained from the appropriate competent authorities and central or local ethics committees as required. Participants provided written informed consent before starting the extension. For those who were illiterate, an independent witness signed the consent form.

Data sharing: The data remain the intellectual property of Imperial College London, but confidential data sharing agreements may be entered into to address the original premise of the trial. Please contact the corresponding author.

1 Lewington S, Clarke R, Qizilbash N, Peto R, Collins R, Prospective Studies Collaboration. Age-specific relevance of usual blood pressure to vascular mortality: a meta-analysis of individual data for one million adults in 61 prospective studies. Lancet 2002;360:1903-13.

2 Guidelines Subcommittee. World Health Organization-International Society of Hypertension Guidelines for the Management of Hypertension. J Hypertens 1999;17:151-83.

3 Mancia G, De Backer G, Dominiczak A, Cifkova R, Fagard R, Germano G, et al. 2007 guidelines for the management of arterial hypertension: the Task Force for the Management of Arterial Hypertension of the European Society of Hypertension (ESH) and of the European Society of Cardiology (ESC). J Hypertens 2007;25:1105-87.

4 Beckett NS, Peters R, Fletcher AE, Staessen JA, Liu L, Dumitrascu D, et al. Treatment of hypertension in patients 80 years of age or older. N Engl J Med 2008;358:1887-98.

5 Peters R, Beckett N, Forette F, Tuomilehto J, Clarke R, Ritchie C, et al. Incident dementia and blood pressure lowering in the Hypertension in the Very Elderly Trial cognitive function assessment (HYVET-COG): a double-blind, placebo controlled trial. Lancet Neurol 2008;7:683-9.

6 Peters R, Beckett N, Burch L, de Vernejoul MC, Liu L, Duggan J, et al. The effect of treatment based on a diuretic (indapamide) + - ACE inhibitor (perindopril) on fractures in the Hypertension in the Very Elderly Trial (HYVET). Age Ageing 2010;39:609-16.

7 Staessen JA, Thijsq L, Fagard R, Celis H, Birkenhager WH, Bulpitt CJ, et al. Effects of immediate versus delayed antihypertensive therapy on outcome in the Systolic Hypertension in Europe Trial. $J$ Hypertens 2004;22:847-57.

8 Bulpitt C, Fletcher A, Beckett N, Coope J, Gil-Extremera B, Forette F, et al. Hypertension in the Very Elderly Trial (HYVET): protocol for the main trial. Drugs Aging 2001;18:151-64.

9 Peters R, Beckett N, Nunes M, Fletcher A, Forette F, Bulpitt C, et al. A substudy protocol of the Hypertension in the Very Elderly Trial assessing cognitive decline and dementia incidence (HYVET-COG): an ongoing randomised, double-blind, placebo-controlled trial. Drugs Aging 2006;23:83-92.

10 Colvez A. Disability free life expectancy. In: Ebrahim S, Kalache A, eds. Epidemiology in old age. BMJ Publishing Group, 1996:41-8.

11 Kostis JB, Wilson AC, Fruedenberger RS. Long term effect of diuretic based therapy on fatal outcomes in subjects with isolated systolic hypertension with and without diabetes. Am J Cardiol 2005;95:29-35.

12 Sutton-Tyrell K, Wildman R, Newman A, Kuller LH. Extent of cardiovascular risk reduction associated with treatment of systolic hypertension. Arch Intern Med 2003;163:2728-31.

Accepted: 05 September 2011

\section{Cite this as: BMJ 2012;344:d7541}

This is an open-access article distributed under the terms of the Creative Commons Attribution Non-commercial License, which permits use, distribution, and reproduction in any medium, provided the original work is properly cited, the use is non commercial and is otherwise in compliance with the license. See: http://creativecommons.org/licenses/bync/2.0/ and http://creativecommons.org/licenses/by-nc/2.0/legalcode. 


\section{Tables}

Table 1 | Characteristics of patients who entered one year extension at start of extension, compared with those who did not enter extension. Values are mean (SD) unless stated otherwise

\begin{tabular}{|c|c|c|c|}
\hline \multirow[b]{2}{*}{ Characteristic } & \multicolumn{2}{|c|}{ Patients who entered extension } & \multirow{2}{*}{$\begin{array}{l}\text { Patients who did not enter extension* } \\
\qquad(n=170)\end{array}$} \\
\hline & Previously on active treatment $(n=924)$ & Previously on placebo $(n=788)$ & \\
\hline Age (years) & $84.9(3.2)$ & $84.6(4.3)$ & $87.4 \dagger(4.0)$ \\
\hline No (\%) female & $562(60.8)$ & $491(62.3)$ & $113(66)$ \\
\hline Sitting blood pressure $(\mathrm{mm} \mathrm{Hg})$ & $145.0 \ddagger / 76.6 \ddagger(17.8 / 10.0)$ & 159.3/80.8 (19.9/10.3) & $142.9 \dagger / 79.8(19.2 / 11.6)$ \\
\hline Standing blood pressure $(\mathrm{mm} \mathrm{Hg})$ & $141.9 \ddagger / 75.8 \ddagger(19.3 / 11.2)$ & $156.2 / 80.2(19.9 / 10.7)$ & $141.4 \uparrow / 78.8(19.0 / 8.5)$ \\
\hline No (\%) with orthostatic hypotension§ & $42(4.5)$ & $51(6.5)$ & $6(4)$ \\
\hline Heart rate (beats per minute) & $74.8(11.5)$ & $74.9(10.8)$ & $75.1(10.6)$ \\
\hline No (\%) current smokers & $64(6.9)$ & $63(8.0)$ & $11(6)$ \\
\hline No (\%) with diabetes & $38(4.1)$ & $30(3.8)$ & $4(2)$ \\
\hline Total cholesterol (mmol/L) & $5.0(1.0)$ & $4.9(1.0)$ & $5.4 \dagger(0.8)$ \\
\hline $\mathrm{HDL}$ cholesterol (mmol/L) & $1.3(0.4)$ & $1.3(0.4)$ & $1.2(0.3)$ \\
\hline Serum creatinine $(\mu \mathrm{mol} / \mathrm{L})$ & $91.3(25.5)$ & $91.2(24.8)$ & $102.8 \dagger(24.8)$ \\
\hline Uric acid $(\mu \mathrm{mol} / \mathrm{L})$ & $299.1 \ddagger(93.4)$ & $274.2(84.6)$ & $254.4(97.7)$ \\
\hline Body mass index $\left(\mathrm{kg} / \mathrm{m}^{2}\right)$ & $23.8(3.8)$ & $23.7(3.9)$ & $24.4(4.4)$ \\
\hline
\end{tabular}

HDL=high density lipoprotein.

${ }^{*}$ Full data not available for 40 patients.

$\dagger$ Significant difference between patients in extension and those not in extension $(P<0.05)$.

$¥$ Significant difference between patients in extension previously on active treatment and those in extension previously on placebo $(P<0.05)$.

§Drop of $>20 \mathrm{~mm} \mathrm{Hg}$ in systolic blood pressure or $>10 \mathrm{~mm} \mathrm{Hg}$ in diastolic blood pressure on standing.

IReported diabetes, taking anti-diabetic treatment, or random blood glucose $>11.1 \mathrm{mmol} / \mathrm{L}$. 
Table 2| Main fatal and fatal plus non-fatal outcomes for intention to treat analysis

Rate per 1000 patient years (No of events)

End points

Previously on placebo treatment Previously on active treatment Hazard ratio* $(95 \% \mathrm{Cl}) \quad$ P value

Fatal and non-fatal stroke

$5.18(4)$

$9.89(9)$

All cause mortality

$38.8(30)$

$18.6(17)$

1.92 (0.59 to 6.22 )

Non-cardiovascular or unknown cause

$14.2(11)$
$6.6(6)$

0.48 (0.26 to 0.87$)$

Cardiovascular mortality

$11.6(9)$

$2.19(2)$

$0.46(0.17$ to 1.25$)$

All heart failure

3.9 (3)

1.1 (1)

$0.19(0.04$ to 0.87$)$

All cardiovascular events $†$

$16.9(13)$

$13.2(12)$

$0.28(0.03$ to 2.73$)$

*Unadjusted.

†Cardiovascular death, stroke, myocardial infarction, and heart failure. 


\section{Figures}

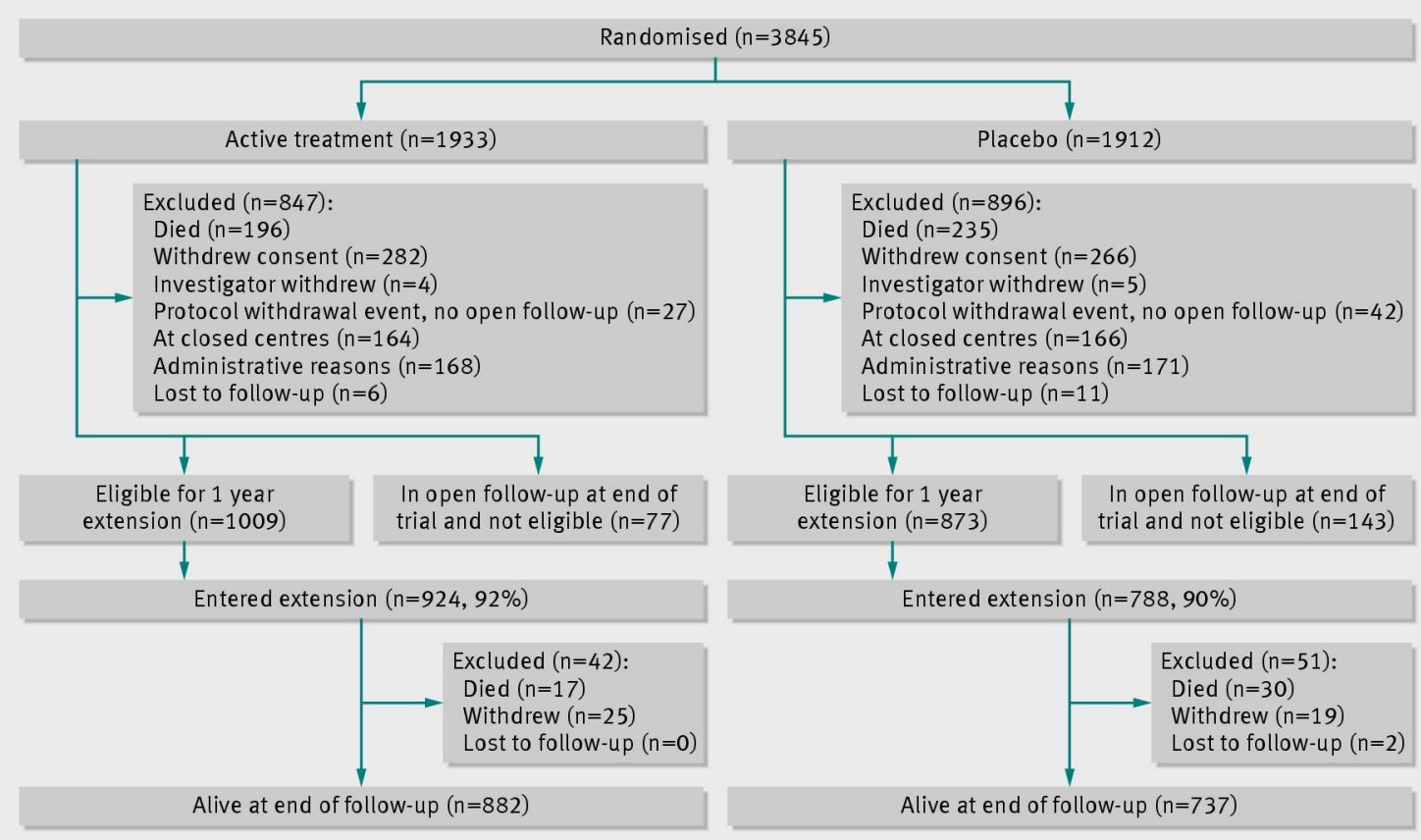

Fig 1 Study profile
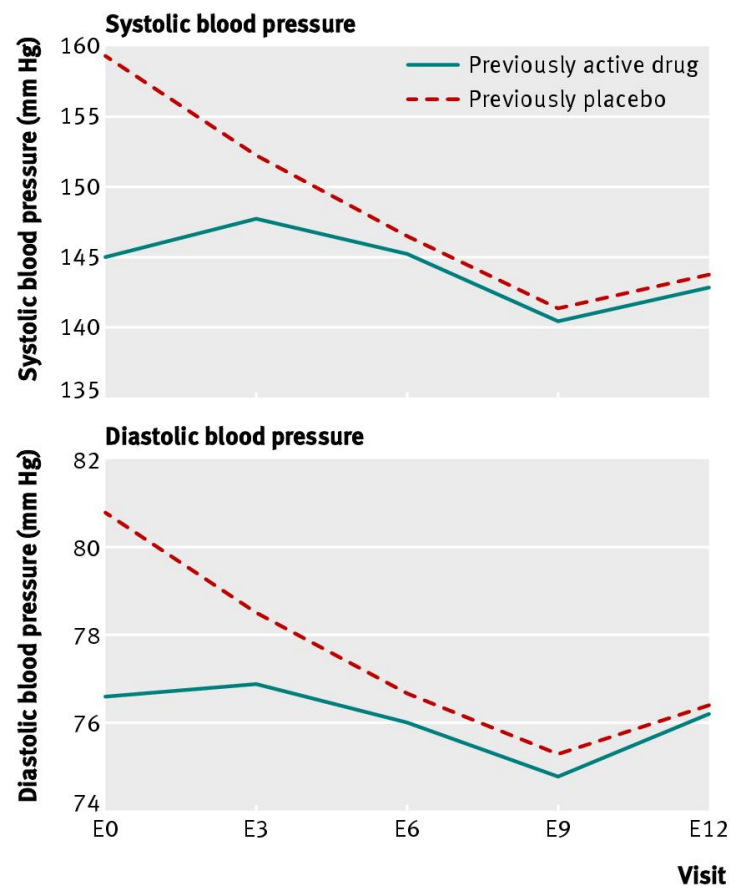

Fig 2 Mean sitting blood pressure by group (previously treated with placebo and previously on active treatment) of participants in extension for systolic blood pressure (top panel) and diastolic blood pressure (bottom panel). E0=visit at start of extension; E3, E6, E12=visits 3, 6, and 12 months after entry into extension 

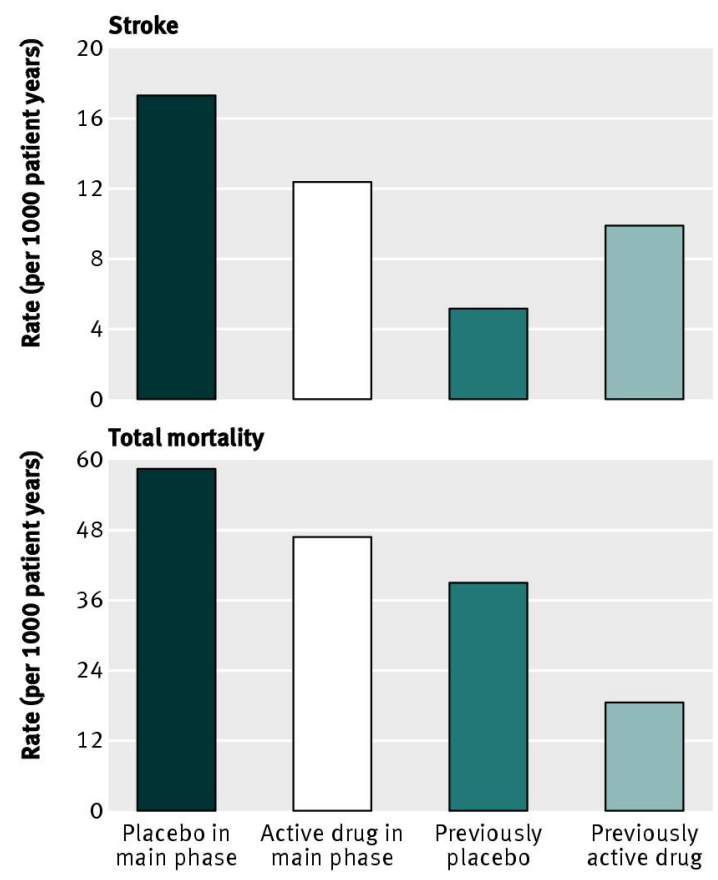

Fig 3 Rates per 1000 patient years in main trial and extension for stroke (top panel) and total mortality (bottom panel) 